\title{
Points of Failure: A Systematic Review of information-flow using Medication Use Cases
}

\author{
Rebecca Hermon \\ Flinders University \\ herm0060@flinders.edu.au
}

\author{
Professor Patricia Williams \\ Flinders University \\ trish.williams@flinders.edu.au
}

\begin{abstract}
Background: Medication errors pose a significant problem in the clinical environment, causing adverse events which impact patient safety.

Problem: The introduction of electronic information and clinical systems have reduced medication errors but have also been identified as creating new types of errors.

Method: Using the previously developed Hermon model, this research aimed to identify and understand medication errors due to clinical information-flow in the Australian General Practice (primary care) setting. The research used existing general practice medication error report cases from the Threat to Patient Safety (TAPS) Study to map against the Hermon model, and validated this mapping through consultations with general practitioners.

Findings: The findings informed the refinement of the Hermon Model, and assisted in identifying medication errors points of information-flow failure in general practice information-flow.

Impact: This study has significance to improve patient safety and inform the development of general practice desktop systems through identification and understanding of information-flow points of failure which result in medication errors.

Keywords: Information-Flow, Information-flow Mapping, Medication Errors, GP Desktop Systems, Threat to Patient Safety (TAPS) Study, Hermon Model
\end{abstract}

\section{Introduction}

Medication errors can cause patient harm and even death. Given medication errors are preventable, it is important to understand and identify them within the medication management information-flow process. Through identifying and understanding the point of failure for information-flow that result in medication errors, it is possible to implement interventions. This research investigated medication errors due to information-flow in the Australian General Practice setting, with particular reference to the use of general practice (GP) desktop systems. Through an information-flow framework called the Hermon model, 164 Threat to Patient Safety (TAPS) cases were mapped to identify medication errors failure points. This enabled the Hermon model to be refined to understand medication errors with reference to GP information-flow and desktop systems. Subsequently, the model underwent an initial validation using consultation with two expect medical practitioners. This paper discusses the background and justification of the research, the method, the Hermon model and the findings from the case study and interview research.

\section{Background}

\section{What are medication errors?}

For the purpose of this research, medication errors have been defined as "an act by a health care professional/ patient that has resulted in a preventable mistake regarding medications that can occur throughout the various stages of the clinical process" [7]. There are several types of medication errors, such as wrong dose, wrong type of medication, missed dose, incorrect patient information and missed medication allergy information [7]. Further, medication errors can occur at defined stages of the patient journey, described as administration, diagnosis, treatment and discharge [7]. Having discussed the types of medication errors that occur, it is important to discuss the causes of these medication errors.

\section{What causes medication errors?}

Traditionally, it has been argued that medication errors result from human errors of many types [5, 12]. Medication errors are often a result of errors of omission and commission by staff or even patients. 
Medication errors can be caused by either lack of knowledge, failure to follow a rule, failure in action such as a slip or failure to recall such as a lapse in memory [5]. According to [2] approximately 33\% of medication errors reported were due to failure to follow policy and procedure or misinterpretation of prescribing information. According to [12] many computer systems may not maintain updated and correct patient medication allergies, resulting in incorrect or potentially harmful prescription medications. Moreover, it is evident that simple human mistakes can be caused by tired, emotional or stressed staff, which can result in medication errors that can have potentially devastating consequences [12]. Historically, messy handwriting has been an immense factor in the interpretation of diagnosis and prescriptions [12] as staff ranging from nurses to doctors can misinterpret a diagnosis or prescription that has been written. Often medications can sound or look similar and confusion can occur, which results in the wrong medication being prescribed. A large number of patients may pressure physicians to quickly diagnose them, and as a result, a patient may receive a vague diagnosis in an aim to be discharged quickly, which can increase the likelihood of a medication error [18]. Additionally, failure to communicate information concerning patient welfare can occur between nurses and doctors, while incorrect staff training, and patient ignorance can also pose a problem. From these errors, it can be seen that medication errors occur within information-flow. This is further supported by [1] who suggested the failure to communicate effectively and efficiently in a timely manner will result in the highly probable occurrence of medication related errors.

\section{The consequences of medication errors}

The consequences of medication errors can have a costly impact to both patients and the healthcare system. For example, [16] estimated that medication errors incur a global cost of $\$ 40$ billion dollars annually. While in Australia alone the cost of medication errors are $\$ 1.4$ billion dollars [11]. Not only do medication errors incur a financial cost but also an impact to patient safety. More specifically $[13,14,15]$ calculated $2 \%$ to $3 \%$ of all hospital admissions were medication error related, while [16] further calculate 7,000 to 9,000 patients die in the United States as a consequence of medication errors. Importantly, medication errors are preventable [12], and through information-flow can be further understood.

\section{Healthcare Information-flow}

Information-flow comes from the theory of information and describes how a piece of information travels from one place to another and its path of transmission [3]. An example of basic information-flow involves understanding how information travels from its original location to its intended recipient. Information-flow requires the "logic of distributed systems" [11, p. 177], which are systems that contain separate parts. Importantly, information systems, and in particular health data systems, are categorised as distributed systems. Health data systems alternatively named health information systems have been defined as "an arrangement of information (data), processes, people, and information technology that interact to collect, process, store and provide as output the information needed to support the health care organization [sic]" [17, p. 105]. Further, GP desktop systems in particular contribute to health data systems. The next section of this paper will discuss GP desktop systems and the relationship to medication errors.

\section{GP Desktop Systems and medication errors}

According to $[6,10] 95 \%$ of General Practitioners in Australia utilise GP desktop systems. Therefore, GP desktop systems play an important role in General Practice, as patient information is collected and stored in these specific health data systems. Building on from the idea that medication errors occur because of human errors; General Practice medication errors are also noted to be a common yet preventable problem. For example, a study conducted by [9] involved the BEACH (Bettering the Evaluation and Care of Health) data collection program. The results indicated that out of 8215 encounters, GPs reported that 852 patients (10.4\%) had experienced adverse drug events, which were caused by a medication error in the previous 6 months. The findings by [9] suggested that $23.2 \%$ of the events that were reported by GP's were preventable. Having discussed medication errors and GP desktop systems within health information systems, it is now necessary to discuss the role of the clinical information-flow framework in identifying and understanding medication errors.

\section{Clinical information-flow framework (Hermon Model)}

Previous research by [7] devised a framework that identified information-flow interruption that results in medication errors. Highlighted in Figure 1 is the Hermon Model, which was created to represent the possibility of miscommunication occurring at any stage of the clinical information process. Highlighted in 
Figure 1, the Hermon model depicts the outcome, event and original error that caused the medication error. It should be noted that the process definition of information-flow errors did not include other errors in the clinical process that may have occurred. The Hermon Model represents information-flow failure in the clinical process to demonstrate and identify the point in the process where errors occur and how this relates to communication.

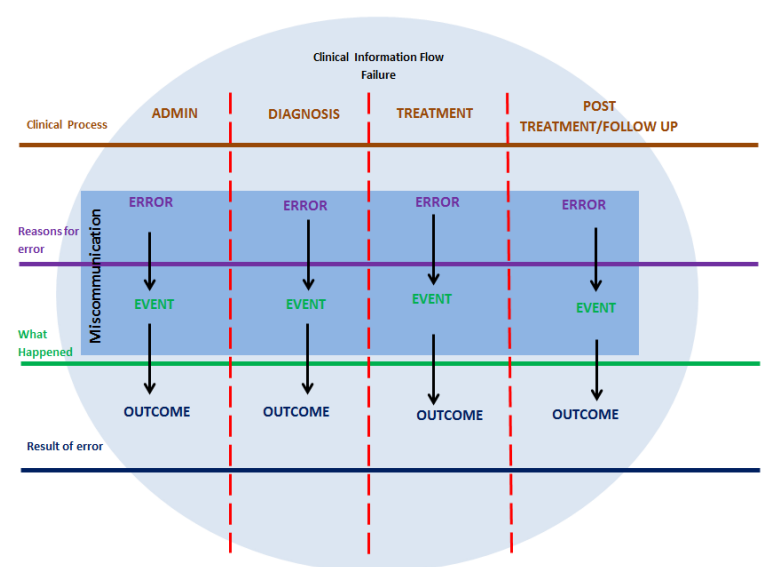

Figure 1. The original Hermon Model developed to understand medication errors [7]

This research focussed on how the generalised Hermon model could be used to analyse GP reported medication information-flow incidents.

\section{Method}

The aim of the project was to conduct an investigation into the prevention of medication errors due to information-flow within the general practice environment. To achieve this objective the following steps in research were taken:

1. Determine whether the previously devised Hermon Model could be used to identify general practice medication errors due to information-flow failure.

2. Identify information-flow failure in GP desktop systems.

3. Recommend possible solutions and future research that would apply the clinical information-flow model.

This research looked at information-flow and points of information-flow failure, as such information systems theory research was the methodology of this research project. However, case study and interview methods were deployed to answer the following research question.

\section{Research Question:}

How can the Hermon Model be used to identify and understand medication errors due to clinical information-flow in Australian General Practice?

\section{Case Study:}

The case study aimed to build on the existing clinical information-flow (Hermon) model through mapping of known cases to the Hermon Model. The mapping of the cases was initially completed by the primary researcher and then confirmed by expert researchers in the group. The mapping of cases was required to validate the Hermon model and identify the points in which medication errors occur in informationflow. The Threat to Patient Safety (TAPS) Study [8] which had been previously conducted by Meredith Makeham, determined the rate of errors reported by GPs in the State of New South Wales, Australia through anonymous reporting of errors using a secure web-based questionnaire. The TAPS study, resulted in 418 error reports by General Practitioners. Medication error cases were set as the inclusion criteria and resulted in the identification of 164 TAPS case studies that could be mapped against the Hermon model. As shown in Table 1 the case studies were categorized into the four categories that comprise the clinical process (Admin, Diagnosis, Treatment and Post Treatment/Follow-Up) in order to evaluate the type and number of errors mapped. The categories of the clinical process are defined below:

Admin: Administration involves, but is not limited to, administration work, medication history/ patient history, clinical records and documentation, results, computer systems.

Diagnosis: The category diagnosis is distinguished by events in which patients are diagnosed.

Treatment: This category refers to decision support provided by GP desktop systems.

Post Treatment/Follow Up: this category refers to events which occur after the patient has been diagnosed and treated. Most often, this refers to events in which the patient is at home.

\section{Results}


As can be seen from Table 1, 19/164 cases were errors that started in Admin, 3/164 started in diagnosis, 132/164 started in Treatment and 10/164 started in the Post Treatment/Follow Up category. The results in Table 1 show that the majority of errors involve the Treatment category which ranges from prescribing medications to dispensing.

Table 1. Where TAPS cases fit within the clinical process points.

\begin{tabular}{cc}
\hline Clinical Process Points & Total Case Studies \\
\hline Admin & 19 \\
Diagnosis & 3 \\
Treatment & 132 \\
Post Treatment & 10 \\
Total & 164 \\
\hline
\end{tabular}

Table 2 identifies the course of the error. What stands out in the table is that 91 errors were initiated by the GP. Additionally, 29 cases were found to be the result of a pharmaceutical error, clinicians were to have caused 13 errors, while hospital staff had caused 9 errors, and nursing home staff were responsible for 6 error cases. It should be noted that further analysis of error cases reveal that several factors can contribute to a medication error and many individuals or factors may be responsible at the time of the error.

Table 2. Source of error

\begin{tabular}{rr}
\hline Who caused the error & Total Case Studies \\
\hline GP & 91 \\
Pharmacist & 29 \\
Locum & 1 \\
Specialist & 2 \\
RMO & 1 \\
Clinician & 13 \\
Hospital & 9 \\
Radiology & 1 \\
Nursing Home & 6 \\
Nurse & 2 \\
Pharmaceutical company & 1 \\
Reception & 3 \\
Patient & 5 \\
Total & 164 \\
\hline
\end{tabular}

Table 3 provides the reasons for the medication error and Table 4 identifies the type of medication error.3

Table 3. What was the error?

\begin{tabular}{cc}
\hline Reason & Total Case Studies \\
\hline Communication Failure & 16 \\
Malpractice & 2 \\
Medication error & 71 \\
Misfiled Notes & 1 \\
Failure to follow up & 4 \\
Recall & 1 \\
Misinformed/ uniformed GP & 16 \\
Wrong Diagnosis & 1 \\
Wrong Patient & 1
\end{tabular}

Table 4 demonstrates that miscommunication was the reason for errors at 16 cases. While errors during the process of prescribing and dispensing medication resulted in 71 error cases. Uniformed or misinformed GPs were also a factor in 16 cases. Other reasons included misfiled notes, failure to follow up, recall, wrong diagnosis or wrong patient. Classification of the various causes of errors show that communication between GPs and patients resulted in 5 error cases. Medication errors that held the highest errors involve the areas of wrong medication dosage (18 cases) and medication management (19 cases).

\section{Table 4. Types of medication errors}

\begin{tabular}{lc}
\hline \multicolumn{1}{c}{ Medication error type } & Total Case Studies \\
\hline Wrong medication dosage & 18 \\
Wrong medication type & 11 \\
Wrong dosage instructions & 1 \\
Wrong medication dispensed & 9 \\
Wrong medication on prescription & 11 \\
Medication mismanagement & 19 \\
No medication dispensed & 2 \\
$\quad$ Total & 71 \\
\end{tabular}

Table 5 shows the number of cases which clearly identified human error rather than an information-flow failure. Human error refers to simple human mistakes such as slips/lapse. Lapse refers to a lapse in memory while slips referring to a mistake.

Table 5. Human error

\begin{tabular}{cc}
\hline Reason & Total Case Studies \\
\hline Human error & 26
\end{tabular}

Decision Support from software was placed in a separate category and each case study that involved a computer desktop error was recorded. Table 6 shows that 41 cases were due to computer error. Poor 
discharge summaries (2 cases), incorrect computer prescriptions (12 cases), wrong patient files (16 cases) and not reviewing computer notes ( 3 cases) were some of the error causes recorded. Lack of computer alarms ( 5 cases) was also noted within the cases mapped and other factors such as confusion of software (2 cases) and juxtaposition errors ( 1 case) were evident.

\section{Table 6. GP desktop system errors}

\begin{tabular}{cc}
\hline GP desktop system & Total Case Studies \\
\hline Incorrect discharge summary & 2 \\
Incorrect dosage selected & 6 \\
Wrong dosage instruction & 2 \\
written & \\
Wrong medication selected & 4 \\
Wrong patient file selected & 15 \\
GP did not review patient & 3 \\
notes & 1 \\
GP did not remove old & 1 \\
medications & 2 \\
Juxtaposition error & 1 \\
Lack of alarms & 5 \\
Nurse & 5 \\
Total & 2 \\
Confusion of software use & 41 \\
\hline
\end{tabular}

Table 7 indicates the number of indeterminable case studies. These two cases contained too little medication error information to classify or map against the Hermon model.

\section{Table 7. Indeterminable cases}

\begin{tabular}{lc}
\hline Indeterminable & Total Case Studies \\
\hline Indeterminable & 2 \\
\end{tabular}

Although each case study was categorised into the clinical process points, further analysis of the mapped case studies showed that many of the case studies fit into entirely different categories. The following tables were created to manage the exceptions and categorise the case studies that did not fit into the four clinical process points as shown in Table 8 .

\section{Table 8. Exceptions}

Highlighted in Table 8 a total of 15/164 exceptions were identified within the case studies that were mapped against the Hermon Model. These exceptions refer to case studies which do not necessarily fit the predetermined 4 categories of clinical process points. They refer events in which a near miss occurred resulting in no adverse event (6/15). Exceptions (1/5) also refer to patient non-compliance in which the patient purposely does not follow instructions given by the GP and therefore resulted in a medication error. Exceptions and interminable cases form part of the limitations of the case studies used as they may contain vague information regarding errors. This section analysed the findings of the mapped TAPS cases against the Hermon model. The next section will discuss how the model was validated through the mapping of case studies.

\section{TAPS Mapping Analysis}

The Hermon model was initially designed to identify and understand the points in the informationflow process where medication errors occur. The result of mapping 164 case studies against the Hermon model resulted in validation of the Hermon model for application to the GP environment. This is because the Hermon model could identify the various points of the medication error within information-flow. However, various improvements to the model were required to better define its validity and ability to map medication errors within a GP setting. Firstly, from the mapped case data it is shown that the majority of errors occurred in treatment. This indicates that treatment errors such as wrong prescription, wrong dosage and wrong medications are just some of the many medication errors that are of common occurrence. A significant finding was that medication errors occur across each clinical process point of the Hermon Model. Therefore, an important aspect of redefining the Hermon model is to indicate what type of treatment error had occurred, and whether or not it involved medication management. This was suggested by the researchers as a refinement of the Hermon model as a result of the mapping of the cases that showed that some errors do not involve medication management. However, it has been recognised that the exceptions (Table 8) are often related to information-flow failure and are therefore required to be mapped against the Hermon Model in order to understand informationflow failure. Secondly, within the mapped case studies, computer systems medical records have been placed in the Admin category. However, it was noticed that errors involving GP desktop systems can start in any of the clinical process points. Therefore, if computer

\begin{tabular}{cc}
\hline Exceptions & Total Case Studies \\
\hline Patient non-compliance & 9 \\
Near Miss & 6 \\
Total & 15 \\
\hline
\end{tabular}


system records stay within the category of Admin, it will limit the validity of the Hermon model. In addition, the Admin clinical process point has been seen to be too vague and could potentially result in confusion between the role of admin and patient medication information. Therefore, the Admin category needed to be well defined, and differentiation between computer systems and administration needed to be categorized. Thirdly, analysis of results indicated that GPs, clinicians and pharmacists are the main cause of errors. Therefore, the model needed to be redesigned to suit the General Practice environment with regards to this research. Lastly, changes to the model were necessary in order for the model to be better understood by clinicians.

From the mapping of the case studies against the Hermon model it is evident that the majority of medications errors are caused by information-flow failure and human error. Miscommunication, uniformed/misinformed GPs and the errors within the clinical desktop systems are just some of the many issues that occur within information flow. A category regarding Software needed to be incorporated within the model, as $22 \%$ of errors were associated with GP desktop systems. Human errors were often due to a lapse in memory or an accidently slip. Further, from the case studies many reported lack of computer alerts was a factor in the occurrence of the medication error. This indicates the important potential alarm intervention and whether or not the Hermon model should include alarms and interventions. Additionally, communication between GPs and patients as well as other entities such as pharmacies and hospitals are of great significance. In particular, communication failure between individuals was a cause of medication errors, whilst appropriate communication and double checking of medications resulted in interventions. Subsequently, the importance of reporting medication errors within the GP has been highlighted through the TAPS case study. Through mapping against the Hermon model, identification and understanding of identifying medication errors failure points within GP informationflow have occurred.

\section{Interviews}

The interviews aimed to validate the revised Hermon model through feedback with two general practitioners. During this stage of the research, the clinicians were consulted to validate the Hermon model with regards to information-flow and information system use. The interviewer asked the clinicians several questions after they assessed the clinical information-flow model against mapped TAPS case studies. Questions regarding the effectiveness of the model to accurately reflect their medication management process were asked.

Questions were informed by the case mapping but included some of the following general questions:

1. Does the model effectively map out clinical errors?

2. Does the model identify where the error occurred? Who it affected, how it occurred and what were the consequences?

3. Can this model be used in a variety of clinical settings?

4. Does the model reflect the use of their GP desktop system in terms of the processes used in medication management?

5. Does the model require improvements?

6. Identify where the GP desktop system used impacts medication errors, as identified in the Hermon model.

\section{Analysis of interviews:}

The answers of the clinicians were analysed to validate the model for General Practice and identify potential points of information-flow failure related to GP desktop systems. A significant finding of the research was that the prescribing of medication occurs directly through General Practice software application and utilises medication data bases which; potentially reduce the likelihood of medication spelling error, assist GPs on information regarding medication dosages and allows GPs to make certain default changes to patient medications, which can prevent medication recall errors. To explain, a recall error occurs when a General Practitioner has a lapse in memory regarding the details of prescribing a patient medication. Additionally, GP desktop systems also identify medication allergies within patients and illuminate possible diagnosis which is coded as dangerous medications or interactions. Interestingly, based on the interview results, issues identified with GP software systems refer to lack of medication dosage warnings, alarm fatigue and that allergies of patients must be entered prior prescription of medication. Nonetheless, GP desktop systems overall are a huge benefit and aid General Practitioners in storing large columns of patient information which can be easily recalled when needed. Concerns were expressed about the Hermon models lack of intuitiveness and understandability. Opinions agreed that the Hermon model required the Admin category to be more clearly defined, and that the clinical process must be clearly shown. While the categories of admin, diagnosis, and treatment and post treatment needed to be evaluated to fit as points in the clinical process. Through the consultations it has been decided that the Hermon model can potentially assist in understanding 
the points of failure in information-flow that may result in medication errors. It is also understood that the model captures GP desktop systems in terms of processes used in medication management. Another reported theme was in regards to the computer error reports raised by GPs. In order to map medication errors against the Hermon framework, the GP is required to raise an in-depth medication error report. However, a common view held was that GPs are not necessarily consistent with information reported within a medication error report. To expand, mapping medication errors from inconsistent and insufficient reporting would result in incomplete and possibly inaccurate identification of process points.

One interviewee suggested the inclusion of a fourth column that contains what could be changed to avoid future error, and that diagnosis should be placed within a separate column. While treatment should show drug interactions and post treatment should show allergies and inappropriate medication treatments. Additionally, it was suggested that the reason for error, drug interactions, allergies, dosage, and inappropriate medications for diagnosis should have a separate column in order to understand the process of medications errors. In addition, the human error/computer error category should be removed as most prescriptions are written in a GP desktop system and are caused by human errors. Overall, these results indicate that the Hermon model needs to be redesigned for GP practice user application.

\section{Findings}

The objective of the research was to conduct an investigation into the prevention of medication errors due to information-flow within the general practice environment. Therefore, using a case study method, the Threat to Patient Safety (TAPS) was mapped to the Hermon model in order to map medication errors in the GP environment. One unanticipated finding was that the data gathered revealed that there was a relationship to human error and the General Practitioner, which often resulted in a snowball effect. The snowball effect relates to errors which further continue to make other errors which then grow to cause harm to patient safety. Further, the case study mapping suggested that a General Practitioner is likely to make a mistake using the computer desktop systems during the treatment stage when prescribing a medication to a patient as evident with 41 errors relating to GP desktop systems. As identified by the snowball effect, this error will then go on to produce further errors such as wrong prescription. For example, 31 out of 71 cases resulted in wrong prescription medication and 23 out of the 71 resulted in wrong medication type. Another important finding was that in the event of an intervention such as an alert, a near miss would result. To illustrate, 6 cases out of 164 cases mapped were a near miss. As mentioned in the literature review medication errors can be caused by a variety of factors and can start at any stage of the clinical process because of human error. This was further supported by the findings of the case study information-flow medication error mapping.

From the consultation interviews the Hermon model was redeveloped into Figure 2 which could be applied to the different aspects of the General Practitioner environment. Redesigning of the Hermon model produced Figure 2 which had a GP prescription medication error approach that focused on medication and adverse event alerts. Additionally, future interventions and solutions were also included as an outcome of the interviews. An outcome of this study was that the model had the ability to map out medication errors which would result in the identification and understanding of these errors.

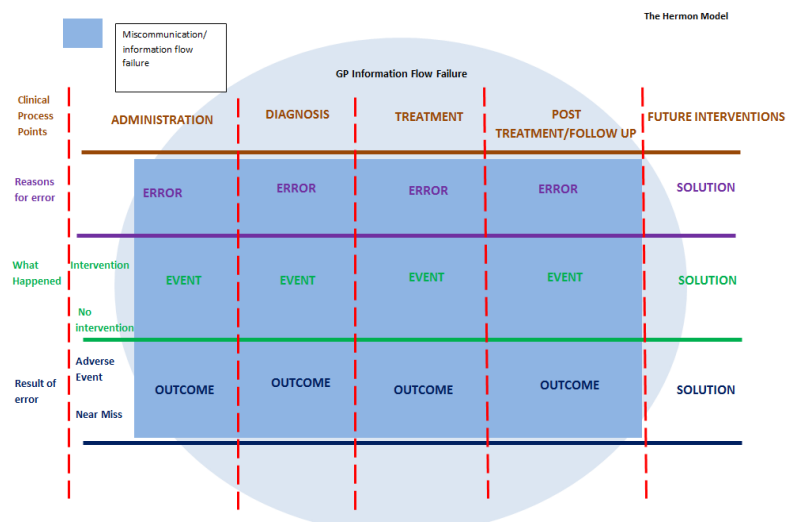

Figure 2. Hermon Model redesigned from TAPS mapping and Interviews.

\section{Discussion}

Medication errors pose a significant problem in the clinical environment, causing adverse events which may include side effects, such as death or disability. Such errors are costly, with an annual expenditure of $\$ 350$ million dollars being spent on medication error related admissions. Information and clinical systems have been identified as one area contributing to medication errors both in terms of the use of IT as well as from traditional information and communication flow processes. Computer and human errors can cause a disruption to the process of information-flow and can result in incorrect drug dosage and other types of 
medication errors. Previous research has been able to identify the points in the clinical information-flow process where medication errors, related to information processing have occurred. These points can be generally categorised as Administration, Diagnosis, Treatment and Post Treatment. Using the previously developed Hermon model, this research aimed to identify and understand medication errors due to clinical information-flow in an Australian General Practice setting. The research used existing General Practice medication error report cases from the Threat to Patient Safety (TAPS) Study to map against the Hermon model, and validated this mapping through consultations with General Practitioners. The findings have resulted in the further refinement of the Hermon Model, and assisted in the identification of how medication errors occur within the GP desktop systems. The significance of this research is to improve patient safety, to inform the development of GP desktop systems. Through this identification of factors, prevention and possible solutions can be developed to prevent medication errors and improve patient safety.

\section{Conclusion}

This study set out to identify, and understand, information-flow related medication errors within GP settings. Therefore, the research question: How can the Hermon model be used to identify and understand medication errors due to clinical information flow failure, originating in Australian General Practice? was posed. Through Case Study analysis and interviews with practitioners the Hermon model was validated and refined. Importantly, this framework was identified to have the capacity to map medication errors within General Practice, which can ultimately allow an improved understanding of medication errors. Whilst this is one clinical example, using a General Practice setting, the model could potentially be used in medication error and incident reporting analysis in other healthcare environments. The main outcome of this research was demonstration of the medication mapping to the Hermon model and to inform its refinement. This demonstrated its ability to assist in understanding and identifying the points of information-flow failure which can result in medication errors. This is important to understand and potentially prevent medication errors associated with the use of GP desktop systems. This research contributes to ongoing research into the safety of health software by identifying points of informationflow failure in medication management processes. The research is unique and has contributed to knowledge because the mapping of medication errors informationflow has not been undertaken previously.

\section{Limitations of Research method chosen}

\section{Using cases}

Interpretation of analysis may be bias or incorrect. In addition, the amount of case studies must be until saturation is evident. Saturation was used to develop enough information to present evidence regarding the Hermon Model. Time constraints are evident, as the analysis of the case studies were time consuming. Additionally, the TAPS cases were conducted prior to 2006, in which there have been several changes to IT.

\section{Interviews}

The participants were appropriately chosen as clinicians who were knowledgeable in e-health and medication errors. Cross checking occurred to prevent personal bias of the Hermon Model. The interview questions were reviewed to assess their relation to the research question, in order to obtain the objectives of the study. During the interview too broad or too narrow questions could have resulted in bias of results [4]. Additionally, a limitation of the interviews was the small validation group (2 GPs).

\section{Ethical considerations}

Various ethical issues were considered during data collection and analysis. Factors such as informed consent, confidentiality and the risks associated with the study were regarded. Individuals participating in interviews were required to be informed of the study and written consent obtained [4], resulting in the approval of ethics for use of the TAPS cases and interviews. Whilst patient information was not being sought, the researcher was careful to protect the confidentiality of any information advertently provided during the consultations of both participants.

\section{Future research}

The next stage of this research would be to further investigate the application of the Hermon model to health information-flow and health systems, in order to identify the aspects that could be addressed in software, and those that could be addressed through education and human interaction.

\section{References}

[1] Commission., E. (2006). Medical Errors. Europe.

[2] Department of Health. (2012-2015). Medication Safety and Opeartional Plan for WA Health: Office of Safety and 
Quatlity in Healthcare. Government of Western Australia, Perth.

[3] Bremer, M. (2004). Information and Information Flow An Introduction. Berlin: Berlin: De Gruyter.

[4] Creswell, J. W. (2013). Qualitative inquiry \&amp; research design: choosing among five approaches (3rd edition. ed.): Thousand Oaks, California: Sage Publications, Inc.

[5] Duguid, M. (2012). The importance of medication reconciliation for patients and practitioners. Aust. Prescr., 35(1), 15-19. doi:10.18773/austprescr.2012.007

[6] Henderson, J., Pollack, A., Gordon, J., \& Miller, G. (2014). Technology in practice - GP computer use by age. Australian Family Physician, 43, 831-831.

[7] Hermon, R. J., \& Williams, P. A. (2013). A study on information induced medication errors. Retrieved from WorldCat.org database. Edith Cowan University, Research Online, Perth, Western Australia.

[8] Makeham, M. A. B., Kidd, M. R., Saltman, D. C., Mira, M., Bridges-Webb, C., Cooper, C., \& Stromer, S. (2006). The Threats to Australian Patient Safety (TAPS) study: incidence of reported errors in general practice. Medical Journal of Australia, 185(2), 95-98. doi:10.5694/j.13265377.2006.tb00482.x

[9] Miller, G. C., Britt, H. C., \& Valenti, L. (2006). Adverse drug events in general practice patients in Australia. Med J Aust, 184(7), 321-324. doi:10.5694/j.13265377.2006.tb00263.x

[10] Miller, R. A., Gardner, R. M., Johnson, K. B., \& Hripcsak, G. (2005). Clinical Decision Support and Electronic Prescribing Systems. Journal of the American Medical Informatics Association, 12(4), 403-409. doi:10.1197/jamia.M1830

[11] Pharmaceutical Society of Australia 2019. Medicine Safety: Take Care. Canberra: PSA.

[12] Rogers, A. (2011). Medical Errors Prevention. In. Miami: University of Miami Miller School of Medicine.

[13] Roughead, E. E., \& Semple, S. J. (2009). Medication safety in acute care in Australia: where are we now? Part 1: a review of the extent and causes of medication problems 2002-2008. In Aust New Zealand Health Policy (Vol. 6, pp. 18).

[14] Roughead, E. E., Semple, S. J., \& Rosenfeld, E. (2016). The extent of medication errors and adverse drug reactions throughout the patient journey in acute care in Australia. International journal of evidence-based healthcare, 14(3), 113. doi:10.1097/XEB.0000000000000075
[15] Roughead, L., Semple, S., \& Rosenfeld, E. (2013). Literature review: medication safety in Australia. Australian Commission on Safety and Quality in Health Care.

[16] Scherbak., R. A. T. Y. (2019). Medication Errors. Retrieved

from https://www.ncbi.nlm.nih.gov/books/NBK519065/

[17] Wager, K. A., Lee, F. W., \& Glaser, J. P. (2013). Health Care Information Systems : A Practical Approach for Health Care Management. Somerset: Somerset: John Wiley \&amp; Sons, Incorporated.

[18] Weingart, S. N., Wilson, R. M., Gibberd, R. W., \& Harrison, B. (2000). Epidemiology of medical error. BMJ, 320(7237), 774-777. 